\section{Robust Kalman Filtering for Discrete Time-Varying Uncertain Systems With Multiplicative Noises}

\author{
Fuwen Yang, Zidong Wang, and Y. S. Hung
}

\begin{abstract}
In this note, a robust finite-horizon Kalman filter is designed for discrete time-varying uncertain systems with both additive and multiplicative noises. The system under consideration is subject to both deterministic and stochastic uncertainties. Sufficient conditions for the filter to guarantee an optimized upper bound on the state estimation error variance for admissible uncertainties are established in terms of two discrete Riccati difference equations. A numerical example is given to show the applicability of the presented method.
\end{abstract}

Index Terms-Additive noise, multiplicative noise, norm-bounded uncertainty, robust Kalman filtering, time-varying system.

\section{INTRODUCTION}

The control and filtering problems for the systems with multiplicative noises have recently received much attention, since the signals contaminated by multiplicative noises are common in many practical systems, such as image processing systems [9], [10], [18], communication systems [20], and aerospace systems (see, e.g., [11] and the references therein). Different from the case of the additive noise, the second order statistics of the multiplicative noise is usually unknown, as it depends on the real state of the system. This gives rise to more difficulties in the research. So far, there have been several approaches to dealing with the control and filtering problems for systems with multiplicative noises, including the game-theoretic method [2], [16], the linear matrix inequality (LMI) approach [1], [3], [4], [21], and the Riccati equation approach [6], to name just a few.

On the other hand, due to unmodeled dynamics, parameter variations, model reduction and linearization, the systems inherently contain the modeling parameter uncertainties. Different kinds of descriptions have been introduced in the literature to account for the uncertainties, such as norm-bounded uncertainty, convex uncertainty, integral quadratic constraint (IQC) uncertainty, linear fractional transformation (LFT) uncertainty. Accordingly, different methods for studying robust control and filtering problems of these uncertain systems have been proposed (see, e.g., [7], [8], [15], [23]-[25], [27], [28], and the references therein). Very recently, Petersen et al. investigated the control problem in [12] and [13] for a class of systems with both stochastic modeling uncertainty and deterministic modeling uncertainty, where the stochastic uncertainty has been expressed as a multiplicative noise. It should be pointed out that, compared to the control case, the corresponding robust filtering problem for systems with stochastic and de-

Manuscript received July 18, 2001; revised January 31, 2002. Recommended by Associate Editor Q. Zhang. The work of F. Yang was supported by the National Natural Science Foundation of China under Grant 69974010. The work of Z. Wang was supported in part by the University of Hong Kong and the Alexander von Humboldt Foundation of Germany. The work of Y. S. Hung was supported by the Research Grants Council of Hong Kong under Grant HKU7043/98E

F. Yang is with the Department of Electrical Engineering, Fuzhou University, Fuzhou 350002, P. R. China (e-mail: fwyang@ @fzu.edu.cn).

Z. Wang was with the Control Theory and Applications Centre, School of Mathematical and Information Sciences, Coventry University, Coventry CV1 5FB, U.K. (e-mail: Zidong.Wang@ @ coventry.ac.uk). He is now with the Department of Information Systems and Computing, Brunel University, Uxbridge, Middlesex UB8 3PH, U.K.

Y. S. Hung is with the Department of Electrical and Electronic Engineering, The University of Hong Kong, Hong Kong (e-mail: yshung@eee.hku.hk).

Publisher Item Identifier 10.1109/TAC.2002.800668. terministic uncertainties has gained much less attention. This situation motivates our present investigation.

In this note, the robust Kalman filtering problem is considered for discrete time-varying systems with two kinds of parameter uncertainties. One is the norm-bounded deterministic uncertainty and the other is the so-called stochastic uncertainty, namely, the multiplicative noise. It is worth emphasizing that the robust Kalman filtering problems have been intensively investigated in the past decade. However, most of the papers have been concerned with the uncertain systems without multiplicative noise (see, e.g., [5], [14], [17], [19], and [26]). In [21] and [22], Wang and Balakrishnan have proposed an LMI method to cope with the stationary robust filtering problem for the uncertain systems with multiplicative noises over an infinite horizon, and a practical example on communication channel filtering problem has been given in [22].

In this note, a robust finite-horizon Kalman filter is designed for the uncertain systems with multiplicative noises where the signals are nonstationary. The problem addressed is the design of a linear filter that yields an estimation error variance with an optimized guaranteed upper bound for all admissible uncertainties. Sufficient conditions for designing such an optimized filter are derived in terms of two discrete Riccati difference equations, which might be suitable for recursive computation in online applications.

The remainder of this note is organized as follows. In Section II, the robust Kalman filtering problem for discrete time-varying systems subject to norm-bounded parameter uncertainty and multiplicative noises is formulated. An algorithm for the filter design is developed in Section III, which guarantees the upper bound on the state estimation error variance and simultaneously minimizes this upper bound. An example is given in Section IV, and some concluding remarks are drawn in Section V.

\section{PROBlem Formulation AND PRELIMINARIES}

Consider the following class of discrete time-varying uncertain systems with multiplicative noises defined on $k \in[0, N]$ :

$$
\begin{aligned}
x_{k+1} & =\left(A_{k}+H_{1, k} F_{k} E_{k}+A_{s, k} \eta_{k}\right) x_{k}+B_{k} w_{k} \\
y_{k} & =\left(C_{k}+H_{2, k} F_{k} E_{k}+C_{s, k} \varsigma_{k}\right) x_{k}+v_{k}
\end{aligned}
$$

where $x_{k} \in R^{n}$ is the state, $y_{k} \in R^{p}$ is the measured output, $w_{k} \in$ $R^{q 1}$ is the process noise, $v_{k} \in R^{q 2}$ is the measurement noise, $\eta_{k} \in R$ and $\varsigma_{k} \in R$ are the multiplicative noises, $A_{k}, A_{s, k}, B_{k}, C_{k}, C_{s, k}$, $H_{1, k}, H_{2, k}$ and $E_{k}$ are known real time-varying matrices with appropriate dimensions, whereas $F_{k} \in R^{i \times j}$ is the norm-bounded timevarying uncertainty, i.e.,

$$
F_{k} F_{k}^{T} \leq I, \quad \forall k .
$$

The parameter uncertainty $F_{k}$ is said to be admissible if it satisfies (2).

The noise signals $w_{k}, v_{k}, \eta_{k}$ and $\varsigma_{k}$ are all Gaussian white noise sequences. They, together with the initial state $x_{0}$, have the following statistical properties:

$$
\begin{gathered}
E\left[w_{k}\right]=0 \quad E\left[v_{k}\right]=0 \quad E\left[\eta_{k}\right]=0 \\
E\left[\varsigma_{k}\right]=0 \quad E\left[x_{0}\right]=\bar{x}_{0} \\
E\left\{\left[\begin{array}{c}
w_{k} \\
v_{k} \\
\eta_{k} \\
\varsigma_{k} \\
x_{0}
\end{array}\right]\left[\begin{array}{c}
w_{j} \\
v_{j} \\
\eta_{j} \\
\varsigma_{j} \\
x_{0}
\end{array}\right]\right\}=\left[\begin{array}{ccccc}
Q_{k} \delta_{k j} & 0 & 0 & 0 & 0 \\
0 & R_{k} \delta_{k j} & 0 & 0 & 0 \\
0 & 0 & \delta_{k j} & 0 & 0 \\
0 & 0 & 0 & \delta_{k j} & 0 \\
0 & 0 & 0 & 0 & S_{0}
\end{array}\right]
\end{gathered}
$$


where $E$ stands for the mathematical expectation operator, $\delta_{k j}$ denotes the Kronecker delta function, which is equal to unity for $k=j$ and zero elsewhere. The known matrices $Q_{k}, R_{k}$, and $S_{0}$ represent the secondorder statistics of the noises and the initial state.

Remark 1: The deterministic uncertainty in $F_{k}$ and the stochastic uncertainties in $\eta_{k}, \varsigma_{k}$ can be scaled and absorbed in the matrices $E_{k}$ and $A_{s, k}, C_{s, k}$, respectively. Therefore, it is reasonable to assume that the deterministic uncertainty in $F_{k}$ satisfies (2) and the stochastic uncertainties in $\eta_{k}, \varsigma_{k}$ satisfy (4). It is worth mentioning that all the noise signals in (1) are modeled as the zero-mean Gaussian white noise sequences. This, however, does not cause any loss of generality, since color noise can be whitened a priori to the zero-mean Gaussian white noise.

Note that the system matrix and output matrix in (1) contain both deterministic parametric uncertainties $H_{1, k} F_{k} E_{k}, H_{2, k} F_{k} E_{k}$ and the stochastic parametric uncertainties $A_{s, k} \eta_{k}, C_{s, k} \varsigma_{k}$, respectively. Due to the complexity in the uncertainties, it is not easy to predict the system states in the form of (1). In the following, we transform (1) into an uncertain system with state-dependent noises:

$$
\begin{aligned}
x_{k+1} & =\left(A_{k}+H_{1, k} F_{k} E_{k}\right) x_{k}+\bar{w}_{k} \\
y_{k} & =\left(C_{k}+H_{2, k} F_{k} E_{k}\right) x_{k}+\bar{v}_{k}
\end{aligned}
$$

where

$$
\bar{w}_{k}=A_{s, k} \eta_{k} x_{k}+B_{k} w_{k} \quad \bar{v}_{k}=C_{s, k} \varsigma_{k} x_{k}+v_{k} .
$$

It can be seen that the noise signals $\bar{w}_{k}$ and $\bar{v}_{k}$ depend on the system state $x_{k}$ and their second-order statistics are unknown.

The statistical properties of the noise signals (6) can be described as follows:

$$
\begin{aligned}
& E\left[\bar{w}_{k}\right]=0, \quad E\left[\bar{v}_{k}\right]=0 \\
& E\left\{\left[\begin{array}{c}
\bar{w}_{k} \\
\bar{v}_{k}
\end{array}\right]\left[\begin{array}{ll}
\bar{w}_{k}^{T} & \bar{v}_{k}^{T}
\end{array}\right]\right\} \\
& =\left[\begin{array}{cc}
A_{s, k} \tilde{P}_{k} A_{s, k}^{T}+B_{k} Q_{k} B_{k}^{T} & 0 \\
0 & C_{s, k} \tilde{P}_{k} C_{s, k}^{T}+R_{k}
\end{array}\right]
\end{aligned}
$$

where

$$
\tilde{P}_{k}:=E\left[x_{k} x_{k}^{T}\right] .
$$

Now, consider the following filter for the uncertain system (5):

$$
\hat{x}_{k+1}=\hat{A}_{k} \hat{x}_{k}+\hat{K}_{k}\left(y_{k}-C_{k} \hat{x}_{k}\right)
$$

where $\hat{x}_{k} \in R^{n}$ is the state estimate, $\hat{A}_{k}$ and $\hat{K}_{k}(0 \leq k \leq N)$ are the filter parameters to be determined.

The objective of this note is twofold. First, we intend to design a finite-horizon filter of the structure (10), such that for all admissible uncertainties in $F_{k}\left(F_{k}\right.$ meets $\left.F_{k} F_{k}^{T} \leq I\right)$, there exist a sequence of positive-definite matrices $\Theta_{k}(0<k \leq N)$ satisfying

$$
E\left[\left(x_{k}-\hat{x}_{k}\right)\left(x_{k}-\hat{x}_{k}\right)^{T}\right] \leq \Theta_{k}, \quad \forall k
$$

That is, the finite upper bound on the state estimation error variance is guaranteed. Second, we shall minimize the bound $\Theta_{k}$ and obtain an optimized filter eventually. This problem will be referred to as a finite-horizon robust Kalman filtering problem. Note that the bound $\Theta_{k}$ is independent of the second order statistics of the system state, i.e., $\tilde{P}_{k}$ defined in (9).

\section{Finite-Horizon Robust Kalman FILTER Design}

In this section, we shall discuss the robust Kalman filter design problem over finite horizon. Define a new state vector

$$
\tilde{x}_{k}=\left[\begin{array}{l}
x_{k} \\
\hat{x}_{k}
\end{array}\right]
$$

then an augmented system follows from the system (5) and the filter (10) that

$$
\tilde{x}_{k+1}=\left(\tilde{A}_{k}+\tilde{H}_{k} F_{k} \tilde{E}_{k}\right) \tilde{x}_{k}+\tilde{B}_{k} d_{k}
$$

where

$$
\begin{aligned}
d_{k} & =\left[\begin{array}{c}
\bar{w}_{k} \\
\bar{v}_{k}
\end{array}\right] \quad \tilde{A}_{k}=\left[\begin{array}{cc}
A_{k} & 0 \\
\hat{K}_{k} C_{k} & \hat{A}_{k}-\hat{K}_{k} C_{k}
\end{array}\right] \\
\tilde{H}_{k} & =\left[\begin{array}{c}
H_{1, k} \\
\hat{K}_{k} H_{2, k}
\end{array}\right] \quad \tilde{E}_{k}=\left[\begin{array}{ll}
E_{k} & 0
\end{array}\right] \\
\tilde{B}_{k} & =\left[\begin{array}{cc}
I & 0 \\
0 & \hat{K}_{k}
\end{array}\right] .
\end{aligned}
$$

Denote the state covariance matrix of the augmented system (13) by

$$
\tilde{\Sigma}_{k}:=E\left[\tilde{x}_{k} \tilde{x}_{k}^{T}\right]=E\left\{\left[\begin{array}{l}
x_{k} \\
\hat{x}_{k}
\end{array}\right]\left[\begin{array}{l}
x_{k} \\
\hat{x}_{k}
\end{array}\right]^{T}\right\} .
$$

According to (13) and (15), the Lyapunov equation that governs the evolution of the covariance matrix $\tilde{\Sigma}_{k}$ can be written as

$$
\tilde{\Sigma}_{k+1}=\left(\tilde{A}_{k}+\tilde{H}_{k} F_{k} \tilde{E}_{k}\right) \tilde{\Sigma}_{k}\left(\tilde{A}_{k}+\tilde{H}_{k} F_{k} \tilde{E}_{k}\right)^{T}+\tilde{B}_{k} \tilde{\Phi}_{k} \tilde{B}_{k}^{T}
$$

where $\tilde{\Phi}_{k}$ is defined in (8). The initial value is $\tilde{\Sigma}_{0}$ and, for later use, we set $\left[\begin{array}{ll}I & 0\end{array}\right] \tilde{\Sigma}_{0}\left[\begin{array}{ll}I & 0\end{array}\right]^{T}=S_{0},\left[\begin{array}{ll}I & -I\end{array}\right] \tilde{\Sigma}_{0}\left[\begin{array}{ll}I & -I\end{array}\right]^{T}=E\left[\left(x_{0}-\right.\right.$ $\left.\left.\hat{x}_{0}\right)\left(x_{0}-\hat{x}_{0}\right)^{T}\right]:=S_{1}$.

It is noted that the uncertainty $F_{k}$ appears in (16). Therefore, it is impossible to give the exact value of the covariance matrix $\tilde{\Sigma}_{k}$. An alternative way is to find a set of upper bounds for $\tilde{\Sigma}_{k}$ and then obtain the minimum with respect to the filter parameters $\hat{A}_{k}$ and $\hat{K}_{k}$.

We now recall some useful lemmas.

Lemma 1 [1]: Let $Y$ be a symmetric matrix, $A, H$ and $E$ be real matrices, $X$ be a symmetric positive-definite matrix and a matrix $F$ satisfy $F F^{T} \leq I$. Then

$$
(A+H F E) X(A+H F E)^{T}-Y \leq 0
$$

if and only if there exists a constant $\alpha>0$ such that

$$
\begin{array}{r}
\alpha^{-1} I-E X E^{T}>0, \\
A\left(X^{-1}-\alpha E^{T} E\right)^{-1} A^{T}+\alpha^{-1} H H^{T}-Y \leq 0 .
\end{array}
$$

Lemma 2 [26]: Given matrices $A, H, E$, and $F$ with compatible dimensions such that $F F^{T} \leq I$. Let $X$ be a symmetric positive-definite matrix and $\alpha>0$ be an arbitrary positive constant such that $\alpha^{-1} I-E X E^{T}>0$, then the following inequality holds

$$
\begin{aligned}
(A+H F E) X(A & +H F E)^{T} \\
& \leq A\left(X^{-1}-\alpha E^{T} E\right)^{-1} A^{T}+\alpha^{-1} H H^{T} .
\end{aligned}
$$

Lemma 3 [19]: For $0 \leq k \leq N$, suppose that $X=X^{T}>0$, $f_{k}(X)=f_{k}^{T}(X) \in R^{n \times n}$ and $g_{k}(X)=g_{k}^{T}(X) \in R^{n \times n}$. If there exists $Y=Y^{T} \geq X$ such that

$$
f_{k}(Y) \geq f_{k}(X)
$$

and

$$
g_{k}(Y) \geq f_{k}(Y)
$$


then the solutions $A_{k}$ and $B_{k}$ to the following difference equations:

$$
A_{k+1}=f_{k}\left(A_{k}\right) \quad B_{k+1}=g_{k}\left(B_{k}\right) \quad A_{0}=B_{0}>0
$$

satisfy $A_{k} \leq B_{k}$.

Remark 2: Lemma 1 is known as the $S$-procedure technique, which is often utilized to convert the inequality involving norm-bounded uncertainty like (17) into an equivalent matrix inequality with an extra scalar parameter $\alpha$. Lemma 2 is a direct result. Lemma 3 will be used to give the upper bound for the covariance matrix $\tilde{\Sigma}_{k}$.

Now, we are in the position to introduce the notions of quadratic filter and identity quadratic filter for the uncertain system (13).

Definition 1 [14], [26]: The filter (10) is said to be a quadratic filter associated with $\Sigma_{k}$ and $P_{k}$ if there exist symmetric positive-definite matrices $\Sigma_{k}$ and $P_{k}(0 \leq k \leq N)$ such that for all admissible uncertainty $F_{k}$ satisfying (2), the following inequality holds:

$$
\begin{aligned}
&\left(\tilde{A}_{k}+\tilde{H}_{k} F_{k} \tilde{E}_{k}\right) \Sigma_{k}\left(\tilde{A}_{k}+\tilde{H}_{k} F_{k} \tilde{E}_{k}\right)^{T} \\
&-\Sigma_{k+1}+\tilde{B}_{k} \Phi_{k} \tilde{B}_{k}^{T} \leq 0
\end{aligned}
$$

where

$$
\Phi_{k}=\left[\begin{array}{cc}
A_{s, k} P_{k} A_{s, k}^{T}+B_{k} Q_{k} B_{k}^{T} & 0 \\
0 & C_{s, k} P_{k} C_{s, k}^{T}+R_{k}
\end{array}\right] .
$$

According to Lemma 1, (24) holds for all admissible uncertainty $F_{k}$ satisfying (2), if and only if there exist a sequence of positive scalars $\alpha_{k}>0$ such that

$$
\begin{array}{r}
\tilde{A}_{k}\left(\Sigma_{k}^{-1}-\alpha_{k} \tilde{E}_{k}^{T} \tilde{E}_{k}\right)^{-1} \tilde{A}_{k}^{T}+\alpha_{k}^{-1} \tilde{H}_{k} \tilde{H}_{k}^{T} \\
-\Sigma_{k+1}+\tilde{B}_{k} \Phi_{k} \tilde{B}_{k}^{T} \leq 0
\end{array}
$$

and

$$
\alpha_{k}^{-1} I-\tilde{E}_{k} \Sigma_{k} \tilde{E}_{k}^{T}>0
$$

Definition 2: The filter (10) is said to be an identity quadratic filter associated with $\Sigma_{k}$ and $P_{k}$ if there exist symmetric positive-definite matrices $\Sigma_{k}$ and $P_{k}(0 \leq k \leq N)$ and positive scalars $\alpha_{k}(0 \leq k \leq$ $N$ ), such that for all admissible uncertainty $F_{k}$ satisfying (2), both the following equation:

$$
\Sigma_{k+1}=\tilde{A}_{k}\left(\Sigma_{k}^{-1}-\alpha_{k} \tilde{E}_{k}^{T} \tilde{E}_{k}\right)^{-1} \tilde{A}_{k}^{T}+\alpha_{k}^{-1} \tilde{H}_{k} \tilde{H}_{k}^{T}+\tilde{B}_{k} \Phi_{k} \tilde{B}_{k}^{T}
$$

and (27) are satisfied, where $\Phi_{k}$ is given in (25).

Based on Definition 2, we have the following conclusion.

Theorem 1: If $\Sigma_{0}=\tilde{\Sigma}_{0}$ and $\tilde{P}_{k} \leq P_{k}$, then

$$
\tilde{\Sigma}_{k} \leq \Sigma_{k}
$$

where $\tilde{\Sigma}_{k}$ and $\Sigma_{k}$ satisfy (16) and (28), respectively.

Proof: $P_{k} \geq \tilde{P}_{k}$ implies that $\Phi_{k} \geq \tilde{\Phi}_{k}$ and $\tilde{B}_{k} \Phi_{k} \tilde{B}_{k}^{T} \geq$ $\tilde{B}_{k} \tilde{\Phi}_{k} \tilde{B}_{k}^{T}$. Thus, (29) follows directly from Lemma 2 and Lemma 3.

Theorem 1 gives an upper bound for the covariance matrix $\tilde{\Sigma}_{k}$ provided that $\tilde{P}_{k} \leq P_{k}$. Next, we shall first find the upper bound $P_{k}$ for $\tilde{P}_{k}$, then construct an upper bound $\Sigma_{k}$ for $\tilde{\Sigma}_{k}$ and select the filter parameters $\hat{A}_{k}$ and $\hat{K}_{k}$ that minimize

$$
\begin{aligned}
\Theta_{k} & :=\left[\begin{array}{ll}
I & -I
\end{array}\right] \Sigma_{k}\left[\begin{array}{ll}
I & -I
\end{array}\right]^{T} \\
& \geq\left[\begin{array}{ll}
I & -I
\end{array}\right] \tilde{\Sigma}_{k}\left[\begin{array}{ll}
I & -I
\end{array}\right]^{T} \\
& =E\left[\left(x_{k}-\hat{x}_{k}\right)\left(x_{k}-\hat{x}_{k}\right)^{T}\right] .
\end{aligned}
$$

Our main results are summarized in the following theorem that provides a constructive approach to designing the identity quadratic filter with optimized upper bound $\Theta_{k}$. For the purpose of clarity, we only give the sketch of the proof.

Theorem 2: Consider (1). Let $\alpha_{k}>0$ be a sequence of positive scalars. If the following two discrete-time Riccati difference equations:

$$
\begin{aligned}
\Theta_{k+1}= & -\left[\alpha_{k}^{-1} H_{1, k} H_{2, k}^{T}+A_{k}\left(\Theta_{k}^{-1}-\alpha_{k} E_{k}^{T} E_{k}\right)^{-1} C_{k}^{T}\right] \\
& \cdot R_{1, k}^{-1}\left[\alpha_{k}^{-1} H_{2, k} H_{1, k}^{T}+C_{k}\left(\Theta_{k}^{-1}-\alpha_{k} E_{k}^{T} E_{k}\right)^{-1} A_{k}^{T}\right] \\
& +A_{k}\left(\Theta_{k}^{-1}-\alpha_{k} E_{k}^{T} E_{k}\right)^{-1} A_{k}^{T}+\alpha_{k}^{-1} H_{1, k} H_{1, k}^{T} \\
& +A_{s, k} P_{k} A_{s, k}^{T}+B_{k} Q_{k} B_{k}^{T}, \quad \Theta_{0}=S_{1}
\end{aligned}
$$

and

$$
\begin{aligned}
P_{k+1}= & \alpha_{k}^{-1} H_{1, k} H_{1, k}^{T}+A_{k}\left(P_{k}^{-1}-\alpha_{k} E_{k}^{T} E_{k}\right)^{-1} A_{k}^{T} \\
& +B_{k} Q_{k} B_{k}^{T}+A_{s, k} P_{k} A_{s, k}^{T}, \quad P_{0}=S_{0}
\end{aligned}
$$

have positive-definite solutions $\Theta_{k}$ and $P_{k}$ such that $\alpha_{k}^{-1} I-$ $E_{k} P_{k} E_{k}^{T}>0$ and $P_{k}>\Theta_{k}$, then an identity quadratic filter (10) with parameters

$$
\begin{aligned}
\hat{A}_{k}= & A_{k}+\left(A_{k}-\hat{K}_{k} C_{k}\right) \Theta_{k} E_{k}^{T} \\
& \cdot\left(\alpha_{k}^{-1} I-E_{k} \Theta_{k} E_{k}^{T}\right)^{-1} E_{k} \\
\hat{K}_{k}= & {\left[\alpha_{k}^{-1} H_{1, k} H_{2, k}^{T}\right.} \\
& \left.\quad+A_{k}\left(\Theta_{k}^{-1}-\alpha_{k} E_{k}^{T} E_{k}\right)^{-1} C_{k}^{T}\right] R_{1, k}^{-1}
\end{aligned}
$$

where

$$
\begin{aligned}
R_{1, k}=\alpha_{k}^{-1} H_{2, k} H_{2, k}^{T}+C_{s, k} & P_{k} C_{s, k}^{T}+R_{k} \\
& +C_{k}\left(\Theta_{k}^{-1}-\alpha_{k} E_{k}^{T} E_{k}\right)^{-1} C_{k}^{T}
\end{aligned}
$$

will be such that the state estimation error variance satisfies boundedness condition

$$
E\left[\left(x_{k}-\hat{x}_{k}\right)\left(x_{k}-\hat{x}_{k}\right)^{T}\right] \leq \Theta_{k}, \forall k .
$$

Moreover, the filter (10) with parameters (33) and (34) minimizes the bound $\Theta_{k}$. have

Proof: First, substituting (14) into (16) and according to (9), we

$$
\begin{aligned}
\tilde{P}_{k+1}= & \left(A_{k}+H_{1, k} F_{k} E_{k}\right) \tilde{P}_{k}\left(A_{k}+H_{1, k} F_{k} E_{k}\right)^{T} \\
& +B_{k} Q_{k} B_{k}^{T}+A_{s, k} \tilde{P}_{k} A_{s, k}^{T}, \\
\tilde{P}_{0}= & S_{0} .
\end{aligned}
$$

Comparing (37) to (32), and according to Lemmas 2 and 3, we conclude that $\tilde{P}_{k} \leq P_{k}$.

Next, suppose that $\Sigma_{k}$ is of the following form [5], [19]:

$$
\Sigma_{k}=\left[\begin{array}{cc}
P_{k} & P_{k}-\Theta_{k} \\
P_{k}-\Theta_{k} & P_{k}-\Theta_{k}
\end{array}\right] .
$$

Substitute (14), (33), (34), and (38) into the right-hand side of (28), and consider the relationships (31) and (32). Then, direct algebraic manipulations show the right-hand side of (28) is given by

$$
\begin{aligned}
\tilde{A}_{k}\left(\Sigma_{k}^{-1}-\alpha_{k} \tilde{E}_{k}^{T} \tilde{E}_{k}\right)^{-1} \tilde{A}_{k}^{T}+\alpha_{k}^{-1} \tilde{H}_{k} \tilde{H}_{k}^{T} & \\
& +\tilde{B}_{k} \Phi_{k} \tilde{B}=\left[\begin{array}{cc}
P_{k+1} & P_{k+1}-\Theta_{k+1} \\
P_{k+1}-\Theta_{k+1} & P_{k+1}-\Theta_{k+1}
\end{array}\right] .
\end{aligned}
$$


This means (38) is a solution to (28). Now, from the initial conditions in (31) and (32), we know that (16) and (28) have the same initial conditions. It then follows from Theorem 1 that $\tilde{\Sigma}_{k} \leq \Sigma_{k}$. Therefore, we have

$$
\begin{aligned}
E\left[\left(x_{k}-\hat{x}_{k}\right)\left(x_{k}-\hat{x}_{k}\right)^{T}\right] & =\left[\begin{array}{ll}
I & -I
\end{array}\right] \tilde{\Sigma}_{k}\left[\begin{array}{ll}
I & -I
\end{array}\right]^{T} \\
& \leq\left[\begin{array}{ll}
I & -I
\end{array}\right] \Sigma_{k}\left[\begin{array}{ll}
I & -I
\end{array}\right]^{T} \\
& =\Theta_{k}, \quad \forall k
\end{aligned}
$$

which implies (36).

Substituting (14) and (38) into (28), we have

$$
\begin{aligned}
\Theta_{k+1}= & {\left[\begin{array}{ll}
I & -I
\end{array}\right] \Sigma_{k+1}\left[\begin{array}{ll}
0 & I
\end{array}\right]^{T} } \\
= & \alpha_{k}^{-1}\left(H_{1, k}-\hat{K}_{k} H_{2, k}\right)\left(H_{1, k}-\hat{K}_{k} H_{2, k}\right)^{T} \\
& +A_{s, k} P_{k} A_{s, k}^{T}+B_{k} Q_{k} B_{k}^{T} \\
& +\hat{K}_{k}\left(C_{s, k} P_{k} C_{s, k}^{T}+R_{k}\right) \hat{K}_{k}^{T} \\
& +\left[\begin{array}{ll}
A_{k}-\hat{K}_{k} C_{k} & \hat{K}_{k} C_{k}-\hat{A}_{k}
\end{array}\right]\left(\Sigma_{k}^{-1}-\alpha_{k} \tilde{E}_{k}^{T} \tilde{E}_{k}\right)^{-1} \\
& \cdot\left[\begin{array}{ll}
A_{k}-\hat{K}_{k} C_{k} & \hat{K}_{k} C_{k}-\hat{A}_{k}
\end{array}\right]^{T} .
\end{aligned}
$$

We take the partial derivatives of $\Theta_{k+1}$ with respect to $\hat{A}_{k}$ and $\hat{K}_{k}$ as follows:

$$
\begin{aligned}
& \frac{\partial \Theta_{k+1}}{\partial \hat{A}_{k}}=\left[\begin{array}{ll}
A_{k}-\hat{K}_{k} C_{k} & \hat{K}_{k} C_{k}-\hat{A}_{k}
\end{array}\right] \\
& \cdot\left(\Sigma_{k}^{-1}-\alpha_{k} \tilde{E}_{k}^{T} \tilde{E}_{k}\right)^{-1}\left[\begin{array}{ll}
0 & -I
\end{array}\right]^{T}=0 \\
& \frac{\partial \Theta_{k+1}}{\partial \hat{K}_{k}}=-2 \alpha_{k}^{-1}\left(H_{1, k}-\hat{K}_{k} H_{2, k}\right) H_{2, k}^{T} \\
& +2 \hat{K}_{k}\left(C_{s, k} P_{k} C_{s, k}^{T}+R_{k}\right) \\
& +2\left[\begin{array}{ll}
A_{k}-\hat{K}_{k} C_{k} & \hat{K}_{k} C_{k}-\hat{A}_{k}
\end{array}\right] \\
& \text { - }\left(\Sigma_{k}^{-1}-\alpha_{k} \tilde{E}_{k}^{T} \tilde{E}_{k}\right)^{-1}\left[\begin{array}{ll}
-C_{k} & C_{k}
\end{array}\right]^{T}=0 .
\end{aligned}
$$

From (42) and (43), and through tedious but straightforward algebraic manipulation, we obtain the optimal filter parameters $\hat{A}_{k}$ and $\hat{K}_{k}$ as follows:

$$
\begin{aligned}
\hat{A}_{k}= & A_{k}+\left(A_{k}-\hat{K}_{k} C_{k}\right) \Theta_{k} E_{k}^{T} \\
& \cdot\left(\alpha_{k}^{-1} I-E_{k} \Theta_{k} E_{k}^{T}\right)^{-1} E_{k}, \\
\hat{K}_{k}= & {\left[\alpha_{k}^{-1} H_{1, k} H_{2, k}^{T}+A_{k}\right.} \\
& \left.\cdot\left(\Theta_{k}^{-1}-\alpha_{k} E_{k}^{T} E_{k}\right)^{-1} C_{k}^{T}\right] R_{1, k}^{-1} .
\end{aligned}
$$

The filter parameters (44) and (45) are identical to (33) and (34), respectively. Therefore, we can conclude that the filter (10) with parameters (33) and (34) minimizes the bound $\Theta_{k}$. This completes the proof.

Remark 3: Similar structure to $\Sigma_{k}$ in (38) has been used in [5] and [19]. The choice of this special structure has been motivated by arguments related to the minimization of the upper bound of the state estimation error variance. Note that the difference Riccati equations (31) and (32) involve the scalar parameter $\alpha_{k}$. Detailed discussions on the feasibility and convergent properties of such kind of difference Riccati equations can be found in [29] and [30].

\section{A NumERICAL EXAMPLE}

The following uncertain system:

$$
\begin{aligned}
x_{k+1} & =\left[\begin{array}{cc}
0 & -0.5 \\
1 & 1+0.3 \delta_{k}
\end{array}\right] x_{k}+\left[\begin{array}{c}
-6 \\
1
\end{array}\right] w_{k} \\
y_{k} & =\left[\begin{array}{ll}
-100 & 10
\end{array}\right] x_{k}+v_{k}
\end{aligned}
$$

has been considered in several papers [5], [19], [26], where the precise bound on the uncertain parameter $\delta_{k}$ has been assumed to be exactly known, i.e., $\left|\delta_{k}\right| \leq 1$. However, in practice, such a bound can often be

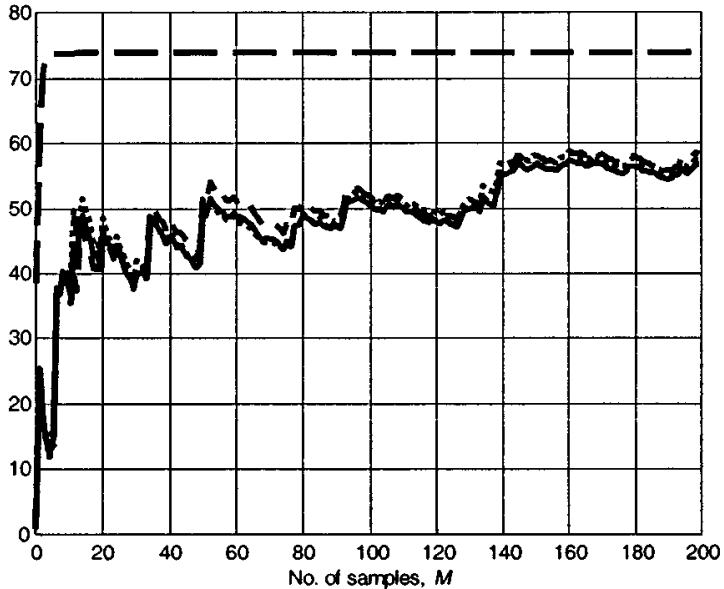

Fig. 1. MSE of the first-state estimate (MSE1) and its upper bound.

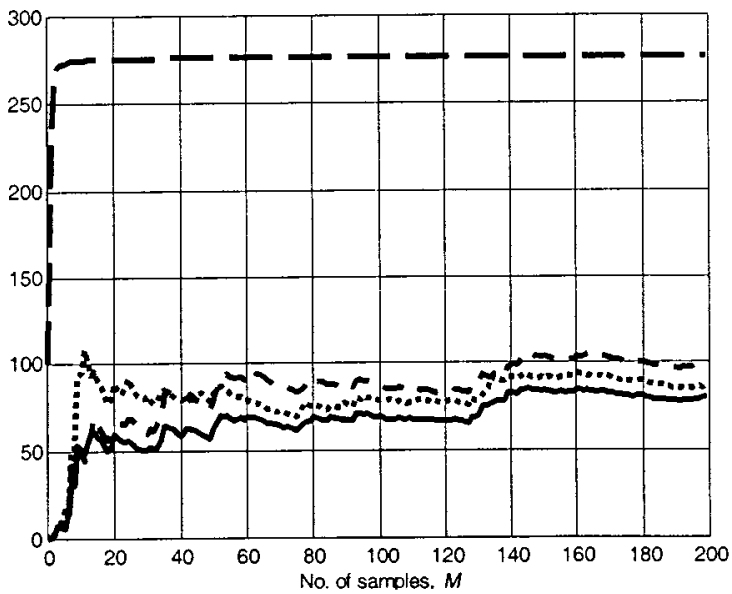

Fig. 2. MSE of the second-state estimate (MSE2) and its upper bound

obtained from the statistical estimation. It is usually the case that the probability of the uncertain parameter $\delta_{k}$ satisfying $\left|\delta_{k}\right| \leq 1$ is close to one, but not equal to one.

In our example, we assume that $P\left(\left|\delta_{k}\right| \leq 1\right)=0.998$ where $P$ means the probability and the statistical law for the uncertain parameter $\delta_{k}$ to satisfy $\left|\delta_{k}\right|>1$ coincides the zero mean Gaussian white noise sequence with intensity 0.1 . This leads to the following uncertain system with multiplicative noise:

$$
\begin{aligned}
& x_{k+1}=\left(\left[\begin{array}{cc}
0 & -0.5 \\
1 & 1+0.3 \bar{\delta}_{k}
\end{array}\right]+\left[\begin{array}{cc}
0 & 0 \\
0 & 0.1
\end{array}\right] \eta_{k}\right) x_{k}+\left[\begin{array}{c}
-6 \\
1
\end{array}\right] w_{k}, \\
& y_{k}=\left[\begin{array}{ll}
-100 & 10
\end{array}\right] x_{k}+v_{k}
\end{aligned}
$$

where the deterministic uncertainty $\bar{\delta}_{k}$ satisfies $\left|\bar{\delta}_{k}\right| \leq 1$ and $w_{k}, v_{k}$ and $\eta_{k}$ are uncorrelated zero mean Gaussian white noise sequences with unity covariance. Note that the system (47) is of the form of system (1) with $H_{1, k}=\left[\begin{array}{ll}0 & 10\end{array}\right]^{T}, H_{2, k}=0$ and $E_{k}=\left[\begin{array}{ll}0 & 0.03\end{array}\right]$. Suppose that the initial conditions are as follows:

$$
x_{0}=\left[\begin{array}{ll}
1 & 0
\end{array}\right]^{T} \quad \hat{x}_{0}=\left[\begin{array}{ll}
0 & 0
\end{array}\right]^{T} \quad S_{0}=100 I_{2} \text { and } S_{1}=10 I_{2} .
$$

The simulations have been conducted with $\alpha_{k}=1.15$ for different deterministic uncertainties $\bar{\delta}_{k}=0, \bar{\delta}_{k}=1$ and $\bar{\delta}_{k}=-1$. Let MSE1 denote the mean square error (MSE) for the estimation of the first state, i.e., $(1 / M) \sum_{k=1}^{M}\left\{\left[\begin{array}{ll}1 & 0\end{array}\right]\left(x_{k}-\hat{x}_{k}\right)\right\}^{2}$, where $M$ is number of samples. Similarly, MSE2 is the mean square error for the estimation of the second state, i.e., $(1 / M) \sum_{k=1}^{M}\left\{\left[\begin{array}{ll}0 & 1\end{array}\right]\left(x_{k}-\hat{x}_{k}\right)\right\}^{2}$. In Fig. 1, the solid (dotted, dashed, respectively) line plots the MSE1 for the case $\bar{\delta}_{k}=0\left(\bar{\delta}_{k}=1, \bar{\delta}_{k}=-1\right.$, respectively). The long dashed line is the 
diagonal element $(1,1)$ of $\Theta_{k}$, which is clearly shown to be the upper bound of MSE1 in all three cases. Analogously, in Fig. 2, the solid (dotted, dashed, respectively) line plots the MSE2 for the case $\bar{\delta}_{k}=0$ $\left(\bar{\delta}_{k}=1,=-1\right.$, respectively), where the long dashed line is the diagonal element $(2,2)$ of $\Theta_{k}$.

We can see from the simulation results that our design goal is well achieved.

\section{CONCLUSION}

A robust finite-horizon Kalman filter has been designed in this note for the uncertain systems with multiplicative noises, which guarantees an upper bound on the state estimation error variance for admissible uncertainties. This bound has been minimized by the construction of filter parameters. Sufficient conditions for a finite-horizon filter to satisfy an upper bound on state estimation error variance for all admissible uncertainties have been given in terms of two discrete Riccati difference equations, which are of a form suitable for recursive computation.

The results obtained have plenty application potentials in many branches of control engineering. For example, in fault detection problems, multiplicative (also called state-dependent) faults could be viewed as a sort of stochastic multiplicative noise uncertainties and our task is to detect multiplicative faults in a finite evaluation window and establish a fault threshold that could be related to the upper bound of the estimation error variance. In such a case, the theoretical results of this note are directly applicable on a real-time basis, and this gives us one of the future research topics.

\section{ACKNOWLEDGMENT}

The authors would like to thank the anonymous reviewers for their insightful comments that helped to improve this note in many aspects.

\section{REFERENCES}

[1] S. Boyd, L. E. Ghaoui, E. Feron, and V. Balakrishnan, Linear Matrix Inequalities in System and Control Theory. Philadelphia, PA: SIAM, 1994, SIAM Studies in Applied Mathematics.

[2] Y. L. Chen and B. S. Chen, "Minimax robust deconvolution filters under stochastic parametric and noise uncertainties," IEEE Trans. Signal Processing, vol. 42, pp. 32-45, Jan. 1994.

[3] V. Dragan and A. Stoica, "A $\gamma$-attenuation problem for discrete-time time-varying stochastic systems with multiplicative noise," in Proc. IEEE Conf. Decision Control, Tampa, FL, 1998, pp. 796-797.

[4] L. El Dhaoui, "State-feedback control of systems with multiplicative noise via linear matrix inequalities," Syst. Control Lett., vol. 24, pp. 223-228, 1995.

[5] M. Fu, C. E. de Souza, and Z. Luo, "Finite horizon robust Kalman filter design," in Proc. IEEE Conf. Decision Control, Phoenix, AZ, 1999, pp. 4555-4560.

[6] E. Gershon, U. Shaked, and I. Yaesh, " $H_{\infty}$ control and filtering of discrete-time stochastic systems with multiplicative noise," Automatica, vol. 37, pp. 409-417, 2001.

[7] M. Green and D. J. N. Limebeer, Linear Robust Control. Upper Saddle River, NJ: Prentice-Hall, 1994.

[8] Y. S. Hung, "Model-matching approach to $H_{\infty}$ filtering," Proc. Inst. Elect. Eng.: Part D, vol. 140, pp. 133-139, 1993.

[9] Y. S. Hung and H. T. Ho, "A Kalman filter approach to direct depth estimation incorporating surface structure," IEEE Trans. Pattern Anal. Machine Intell., vol. 21, pp. 570-575, June 1999.

[10] Y. S. Hung and F. Yang, " $H_{\infty}$ versus Kalman filtering for depth estimation," in Proc. Int. Conf. Signal Processing Intelligent System, Guangzhou, P. R. China, 1999, pp. 276-281.

[11] V. S. Kouikoglou and Y. A. Phillis, "Trace bounds on the covariances of continuous-time systems with multiplicative noise," IEEE Trans. Automat. Contr., vol. 38, pp. 138-142, Jan. 1993.

[12] I. Petersen and M. R. James, "Performance analysis and controller synthesis for nonlinear systems with stochastic uncertainty constraints," $\mathrm{Au}$ tomatica, vol. 32, pp. 959-972, 1996.
[13] I. Petersen, M. R. James, and P. Dupuis, "Minimax optimal control of stochastic uncertain systems with relative entropy constraints," IEEE Trans. Automat. Contr., vol. 45, pp. 398-412, Mar. 2000.

[14] I. Petersen and D. McFarlane, "Optimal guaranteed cost filtering for uncertain discrete-time linear systems," Int. J. Robust Nolinear Control, vol. 6, pp. 267-280, 1996.

[15] I. Petersen and A. V. Savkin, Robust Kalman Filtering for Signals and Systems with Large Uncertainties, ser. Control Engineering. Boston, MA: Birkhauser, 1999.

[16] Y. A. Phillis, "Estimation and control of systems with unknown covariance and multiplicative noise," IEEE Trans. Automat. Contr., vol. 34, pp. 1075-1078, Oct. 1989.

[17] U. Shaked and C. E. de Souza, "Robust minimum variance filtering," IEEE Trans. Signal Processing, vol. 43, pp. 2474-2483, Nov. 1995.

[18] A. M. Tekalp and G. Pavlovic, "Image restoration with multiplicative noise: Incorporating the sensor nonlinearity," IEEE Trans. Signal Processing, vol. 39, pp. 2132-2136, Sept. 1991.

[19] Y. Theodor and U. Shaked, "Robust discrete-time minimum-variance filtering," IEEE Trans. Signal Processing, vol. 44, pp. 181-189, Feb. 1996.

[20] J. Y. Tourneret, M. Coulon, and M. Doisy, "Least-squares estimation of multiple abrupt changes contaminated by multiplicative noise using MCMC," in Proc. IEEE Workshop Higher Order Statistics, 1999, pp. 1148-1152.

[21] F. Wang and V. Balakrishnan, "Robust adaptive Kalman filters for linear time-varying systems with stochastic parametric uncertainties," in Proc. Amer. Control Conf., San Diego, CA, 1999, pp. 440-444.

[22] — "Robust estimators for systems with deterministic and stochastic uncertainties," in Proc. IEEE Conf. Decision Control, Phoenix, AZ, 1999, pp. 1946-1951.

[23] Z. Wang and K. J. Burnham, "Robust filtering for a class of stochastic uncertain nonlinear time-delay systems via exponential state estimation," IEEE Trans. Signal Processing, vol. 49, pp. 794-804, Apr. 2001.

[24] Z. Wang, Z. Guo, and H. Unbehauen, "Robust $H_{2} / H_{\infty}$ state estimation for discrete-time systems with error variance constraints," IEEE Trans. Automat. Contr., vol. 42, pp. 1431-1435, Oct. 1997.

[25] Z. Wang and B. Huang, "Robust $H_{2} / H_{\infty}$ filtering for linear systems with error variance constraints," IEEE Trans. Signal Processing, vol. 48, pp. 2463-2467, Aug. 2000.

[26] L. Xie, Y. C. Soh, and C. E. de Souza, "Robust Kalman filtering for uncertain discrete-time systems," IEEE Trans. Automat. Contr., vol. 39, pp. 1310-1314, June 1994.

[27] F. Yang and Y. S. Hung, "Robust $H_{\infty}$ filtering with error variance constraints for uncertain discrete-time systems," in Proc. IEEE Conf. Control Application, Anchorage, AK, 2000, pp. 635-640.

[28] K. Zhou, J. Doyle, and K. Glover, Robust and Optimal Control. Upper Saddle River, NJ: Prentice-Hall, 1996.

[29] X. Zhu, Y. C. Soh, and L. Xie, "Design and analysis of discrete-time robust Kalman filters," Automatica, vol. 38, no. 6, pp. 1069-1077, 2002.

[30] — "Feasibility and convergence analysis of discrete-time $H_{\infty} a$ priori filters," in Proc. 2000 Amer. Control Conf., 2000, pp. 4179-4183. 